\title{
Simultaneous Fractures of Proximal and Distal End of Radius. An Unusual Pattern of Complex Injuries and Clinical Review
}

\author{
Kastanis G ${ }^{* 1}$, Spyrantis M. ${ }^{1}$, Magarakis G. ${ }^{1}$, Kapsetakis P. ${ }^{1}$, Pantouvaki A. ${ }^{2}$ \\ ${ }^{1}$ Department of Orthopaedic, General Hospital of Heraklion -Venizeleio, Crete, Greece \\ ${ }^{2}$ Department of Physiotherapy, General Hospital of Heraklion -Venizeleio, Crete, Greece \\ *Corresponding Author: Grigorios Kastanis, email: kastanisg@ gmail.com \\ Address: Leoforos Andrea Papandreou 118, Mastambas, Heraklion, 71305, Crete, Greece \\ Received 06 August 2019; \\ Accepted 04 September 2019;
}

Published 26 September 2019

\begin{abstract}
While the isolated fractures of proximal or distal radius are very common injuries in adults and account to $14 \%$ and $18 \%$ of all extremity fractures the simultaneous ipsilateral fractures of proximal and distal end of radius are quite uncommon. We present two cases (females 64 and 56 years old) with ipsilateral fractures of radial head and distal end of radius due to a fall. No signs of ligamentous injuries were found in preoperative magnetic resonance imaging's (MRI). Firstly we treated the distal radius fracture in both cases with volar locking plate, secondly we approached the radial head fracture: in one case (Maison type I) conservatively (plaster of Paris) and in the other case (Maison type III) with radial head replacement. The aim of this study has two objectives: first to increase the awareness of diagnosing this bipolar injury in the emergency department and second to introduce the modalities of treatment.
\end{abstract}

Keyword: Distal radius, Radial head, Simultaneous radial fractures

\section{Introduction}

Distal radius fractures (DRF) are very common injuries and account to $18 \%$ of all fractures in age over 65 years old as a result of low energy force, after a fall from a height with a dorsally outstretched hand. ${ }^{[1]}$ On the other hand radial head fractures account to $14 \%$ of all adults fractures appearing mostly in women over 54 years old when individual falls with extended elbow joint on an outstretched hand. ${ }^{[2]}$ Simultaneous ipsilateral fractures of radial head and distal radius are very uncommon injuries and the treatment guidelines are not described in detail because that depends on various factors (fracture pattern, stability, age, physical demands of the patients).

The aim of this study is to achieve two objectives: The diagnostic deficit to recognize this pattern of complex injuries in the emergency department and to suggest an algorithm of treatment.

\section{Case Report 1}

A woman aged 64 years old was transferred from other medical union to our department with a diagnosed distal radius fracture, eight hours after injury (Fig 1(a) and 1(b)). During clinical examination patient referred, except pain on the left wrist, a mild pain on left elbow. New x-Ray examination (Fig 1(c)) of the left elbow showed a fracture of radial head Maison type I and Ct/Scan of the left wrist showed ipsilateral fracture of distal radius AO/OTA B3 (Fig 2(a),2(b),2(c) and 2(d)). Magnetic resonance imaging showed no ligamentous injury of the interosseous membrane of the forearm complex. The patient was surgically treated, under general anesthesia, for the D.R.F. with extended Flexor Carpi Radialis Approach (Fig 3). Subsequent fixation was done with a volar locking plate (Fig 4(a) and 4(b)). The elbow and wrist were immobilized in an above elbow plaster slab for 2 weeks. Passive motion was performed after two weeks in elbow and wrist joints, using continuous passive motion equipment (CPM) combined with manual therapy, to increase range of movement. A regime of assisted and active range exercises was started in both joints and the patient was taught to follow a daily program of active exercises, ball and putty exercises as well as stretches. Postoperative assessment at three, six months and finally one year showed recovery of range of motion in both joints (wrist joint: dorsal flexion $50^{\circ} / 60^{\circ}$, palmar flexion $70^{\circ} / 80^{\circ}$, radial deviation $20^{\circ} / 20^{\circ}$, ulnar deviation $25^{\circ} / 30^{\circ}$, full supination and pronation of forearm, elbow joint: flexion $130^{\circ} / 135^{\circ}$ and extension $0^{\circ} / 0^{\circ}$ ), with pain-free movements and without loss of grip strength compared to the other hand. Quick Dash score was 2.5 and Cooney's Score was 90. (Fig 5(a), 5(b), 5(c) and 5(d)). 


\section{Figures: Case 1}

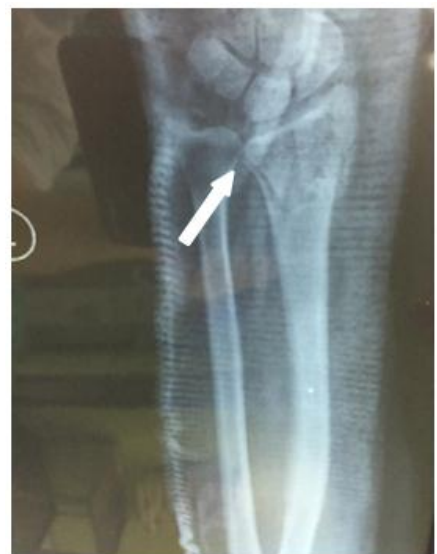

(a)

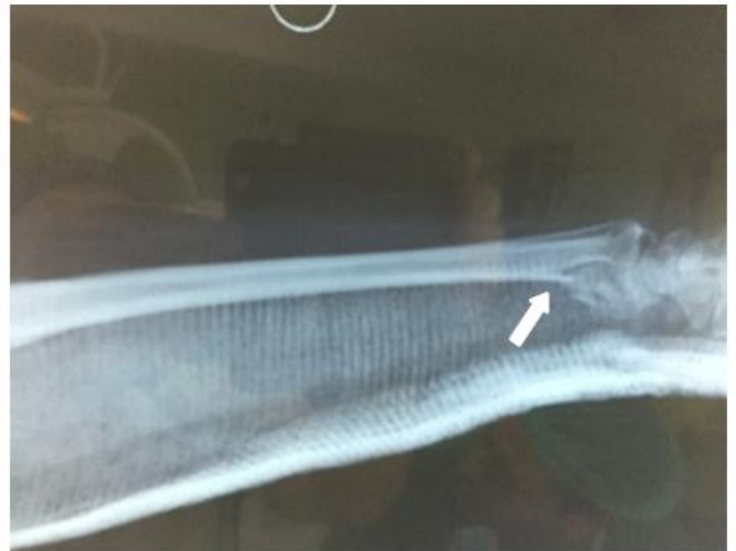

(b)

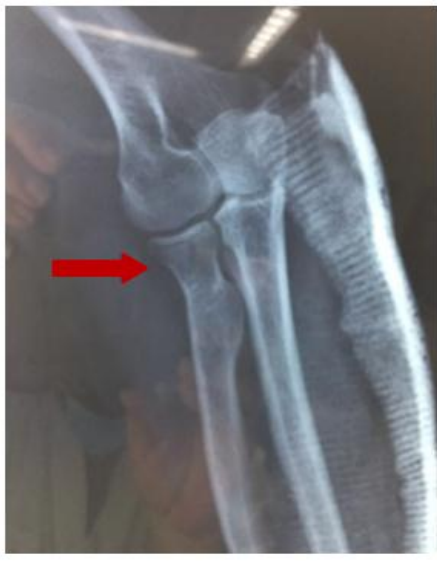

(c)

Case 1: Figure: 1 Preoperative $x$-rays of the left wrist (white arrow) and left elbow (red arrow)

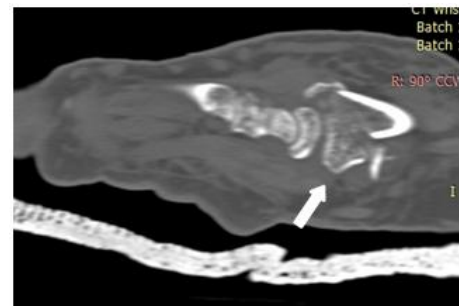

(a)

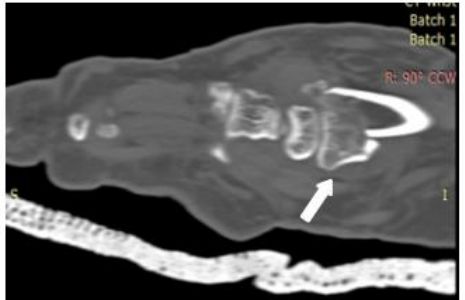

(b)

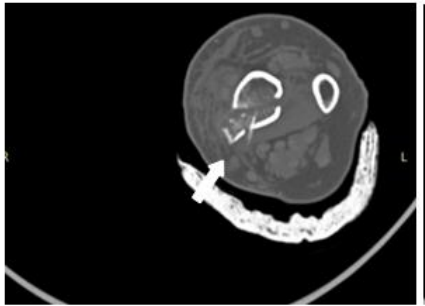

(c)

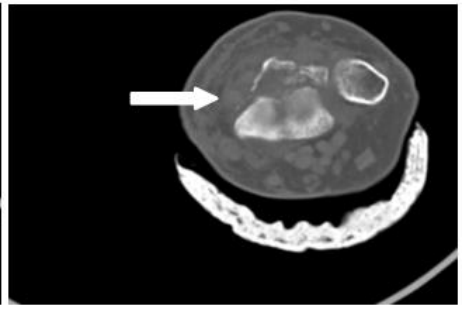

(d)

Case 1: Figure: 2 Preoperative Ct /Scan of the left wrist (white arrow)

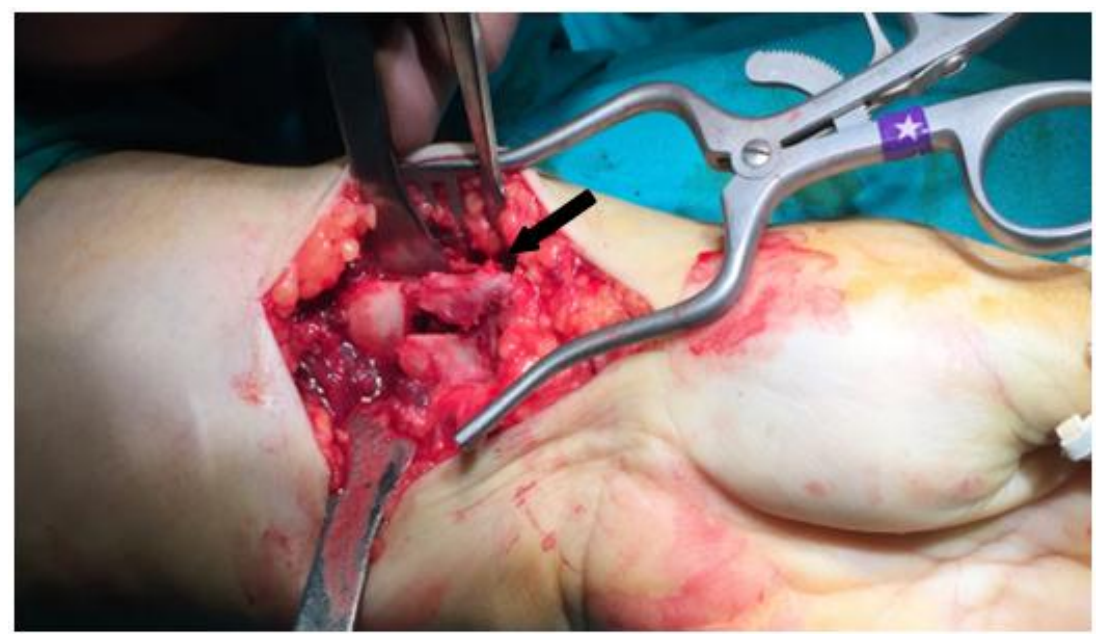

Case 1: Figure: 3 Intraoperative Distal Radius Fracture (black arrow)

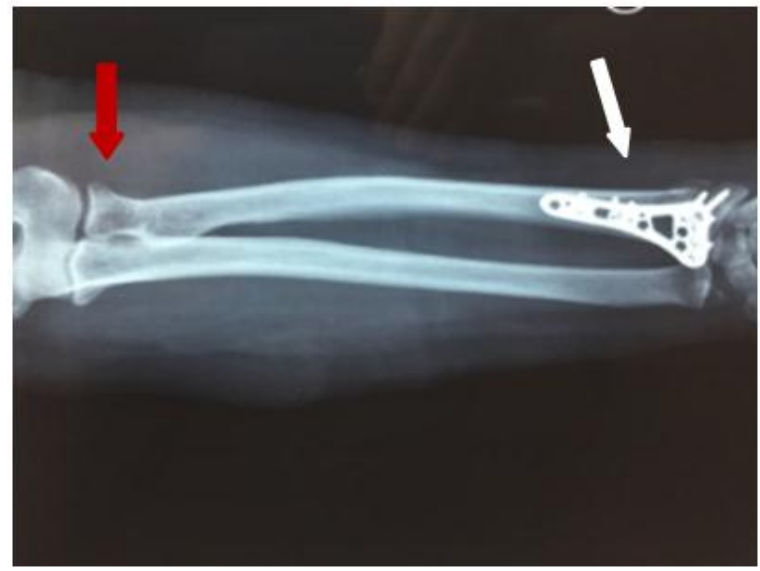

(a)

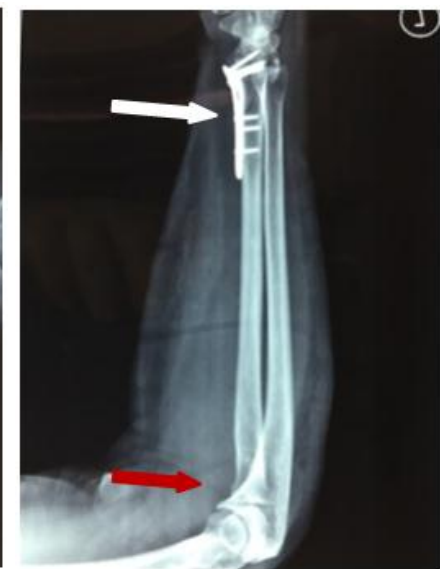

(b)

Case 1: Figure: 4 Postoperative $x$-rays of left elbow (red arrow) and left wrist (white arrow) 


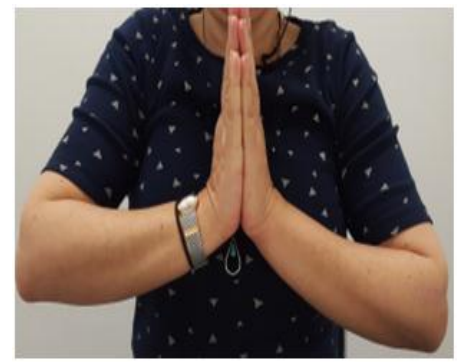

(a)

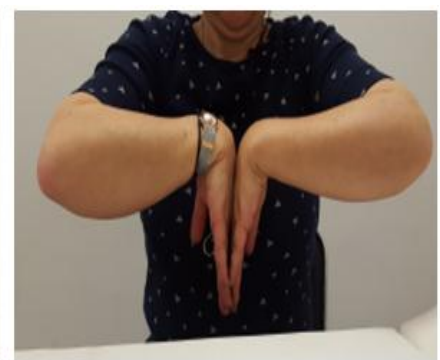

(b)

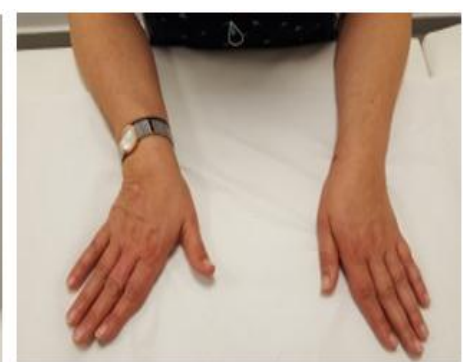

(c)

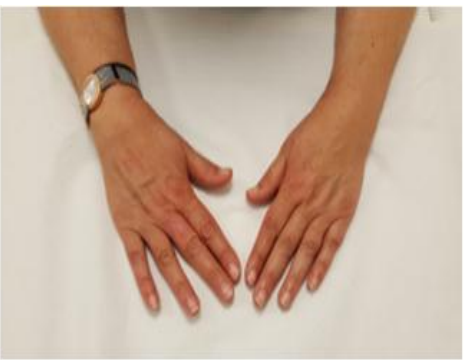

(d)

Case 1: Figure: 5 Range of motion at one year

\section{Case Report 2}

A woman aged 56 years old presented with severe pain on right elbow and wrist, with swelling on radial side of elbow, wrist deformity followed a fall from stairs with a dorsally outstretched hand. Clinical examination showed hematoma with pain and limited range of motion on the right elbow, forearm was held in semi pronation with the elbow in flexion and the wrist in radial deviation. Neurovascular examination of the upper limb was normal. Plain radiographs (Figures 1(a) and 1(b)) and computer tomography (Figures 2(a),2(b),2(c),2(d) and 2(e)) showed comminuted fracture of radial head Maison type III and ipsilateral fracture of distal radius AO/OTA C2 . Preoperative M.R.I. showed no ligamentous injury of the interosseous membrane of the forearm complex. Treatment of this complex injuries starts from the distal radius fracture with scope to restore radial length and to obtain anatomical reduction of intra-articular fragment in order to achieve the correct radial head prosthesis length size and rotation. An extended Flexor Carpi Radialis approach was performed under general anesthesia and after prior reduction with Kirschner wires the fracture was stabilized with a volar locking plate (Figure 3). Then the radial head fracture was treated with Kocher posterolateral approach and excision of the comminuted head (Figure 4). A radial prosthesis was performed and good stability was provided. Control of elbow motion was performed to avoid impingement. Final the ligamentous injury were repaired prior closure. The elbow and wrist were immobilized in an above elbow plaster slab for 2 weeks (Figure 5(a) and 5(b)). Passive motion was performed in elbow and wrist separately using CPM combined with manual therapy to increase range of movement. A regime of assisted and active range exercises was started in both joints and the patient was taught to follow a daily program of active exercises, ball and putty exercises as well as stretches.

Postoperative assessment at 3,6 months and finally at one year showed recovery of range of motion in both joints (wrist joint: dorsal flexion $59^{\circ} / 60^{\circ}$, palmar flexion $85^{\circ} / 90^{\circ}$, radial deviation $25^{\circ} / 30^{\circ}$, ulnar deviation $35^{\circ} / 35^{\circ}$ full supination and pronation of forearm, elbow joint: flexion $135^{\circ} / 140^{\circ}$ and extension $5 \% 0^{\circ}$ ), without pain on movements and without loss of grip strength compared to the other hand. Quick Dash score was 0.0 and Cooney's Score was 95. (Fig 6(a), 6(b), 6(c) and 6(d)).

\section{Figures: Case 2}

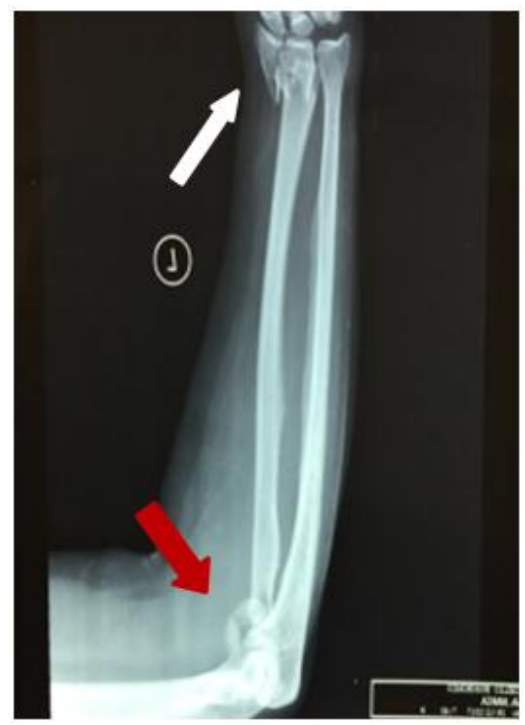

(a)

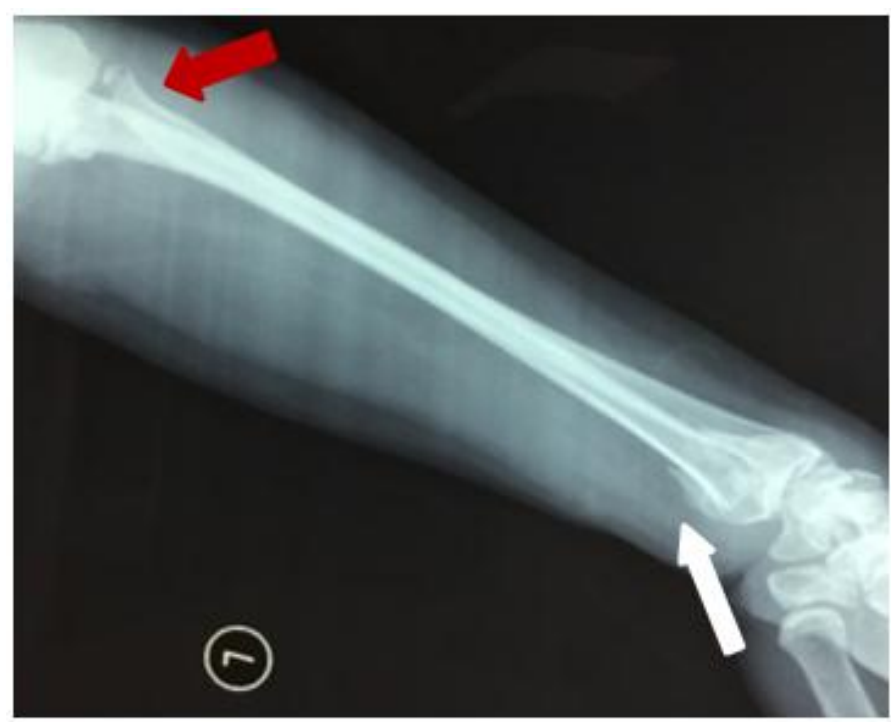

(b)

Case 2: Figure: 1 Preoperative x-rays of the right wrist (white arrow) and elbow (red arrow) 


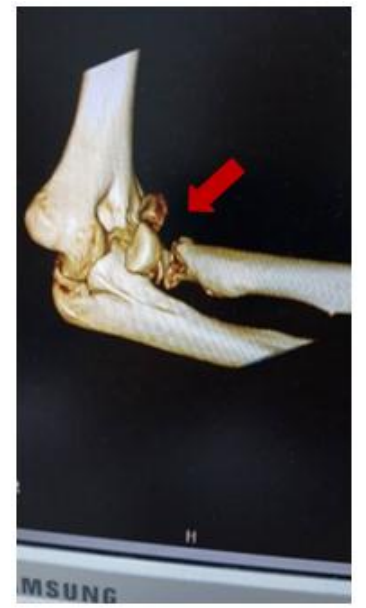

(a)

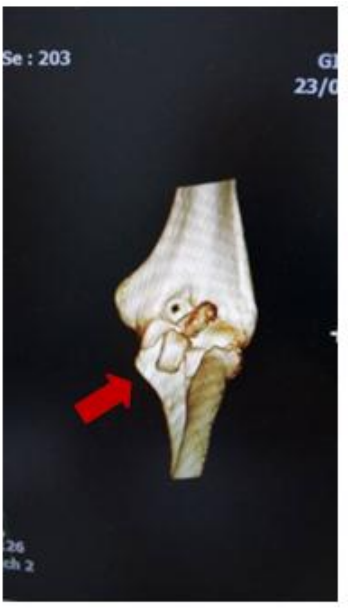

(b)

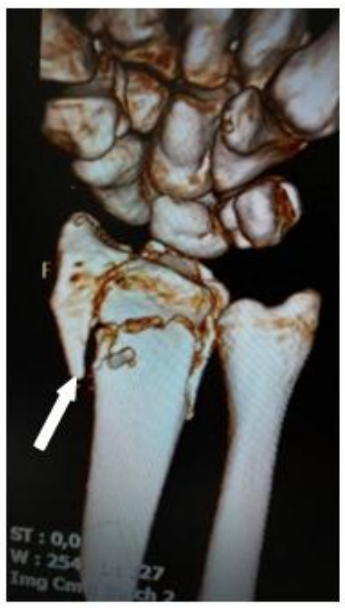

(c)

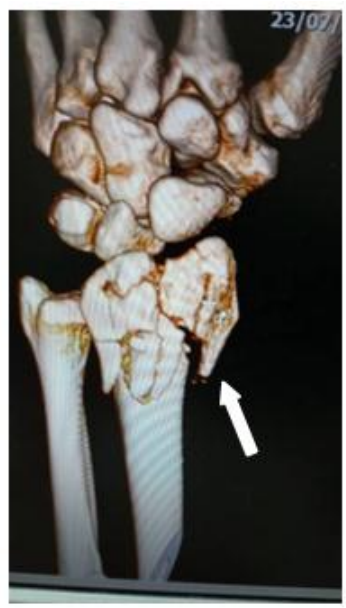

(d)

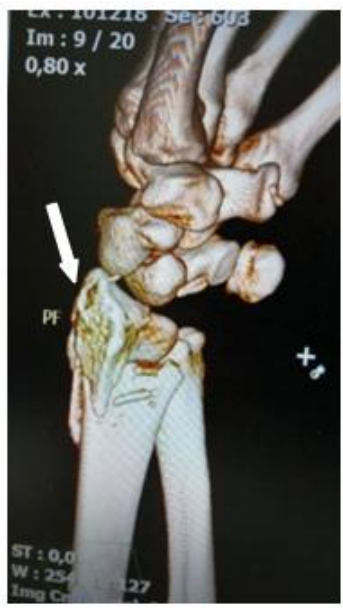

(e)

Case 2: Figure: 2 Preoperative 3D/Scan of the right elbow (a, b red arrow) and the simultaneous right wrist(c, d, e white arrow)

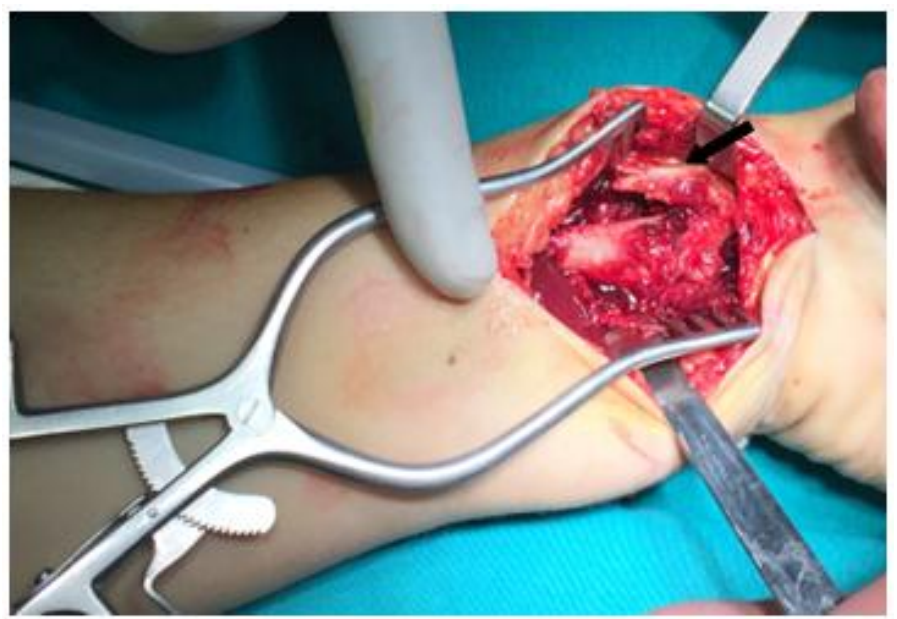

Case 2: Figure: 3 Intraoperative fracture of distal radius (black arrow)

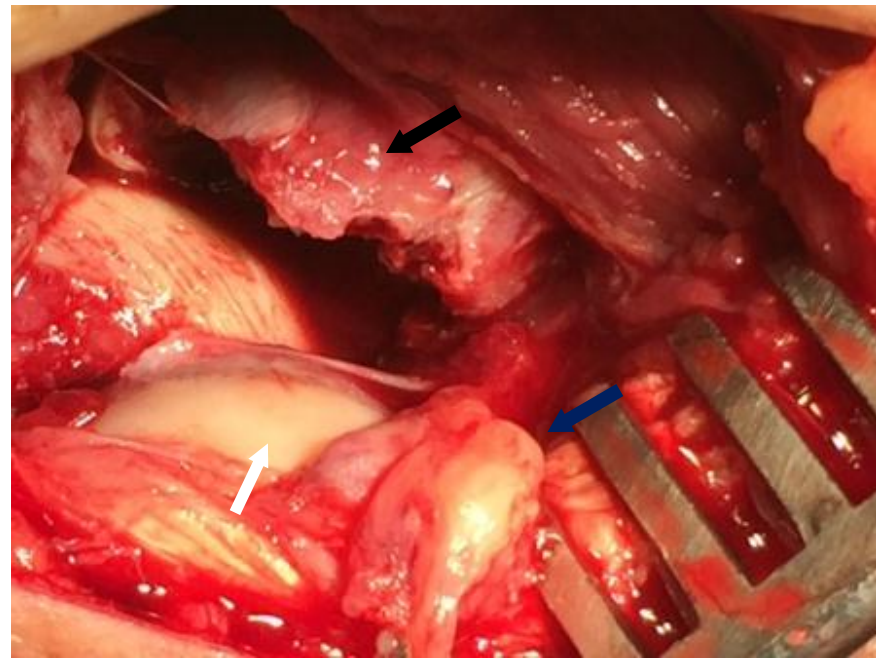

Case 2: Figure: 4 Intraoperative fracture of head of radius (blue arrow), Capitelum (white arrow), radial neck (black arrow) 


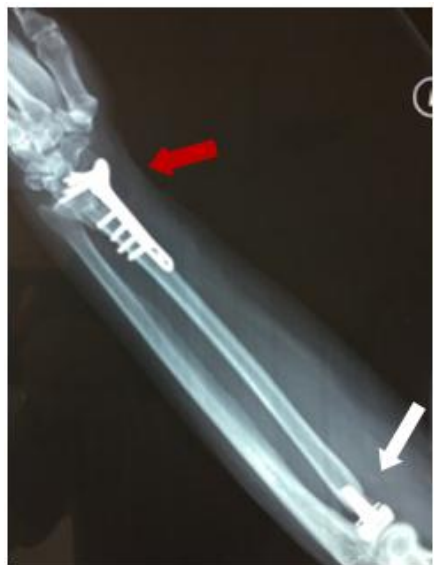

(a)

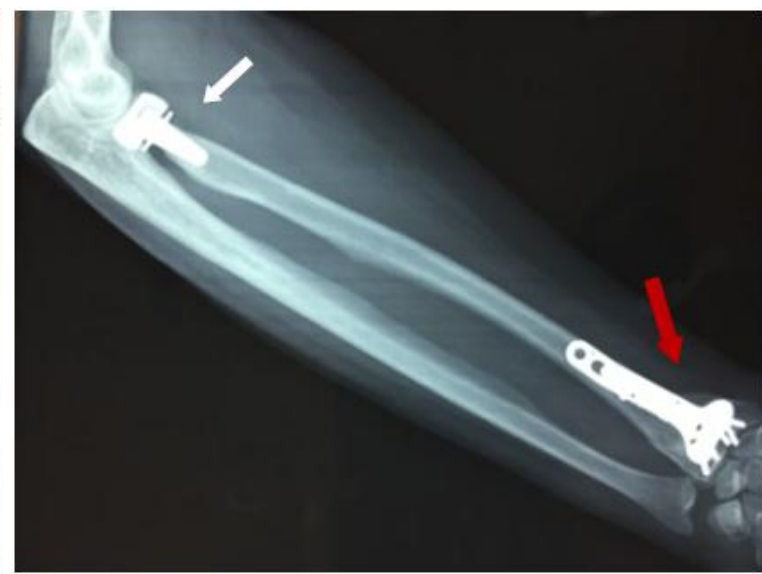

(b)

Case 2: Figure: 5 Postoperative $x$-Rays of the fractures (DRF (red arrow) and radial head implant white arrow)

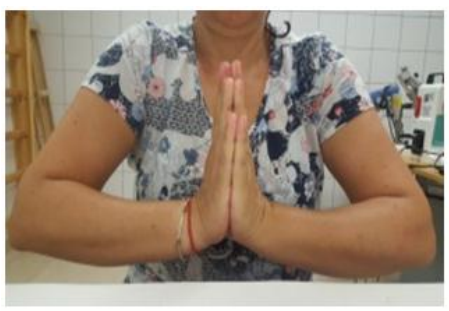

(a)

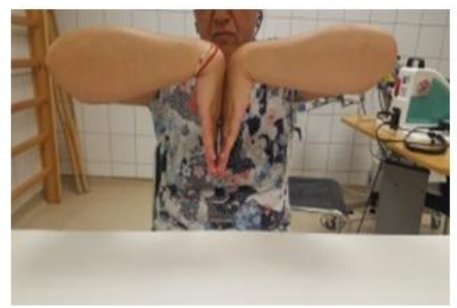

(b)

(c)

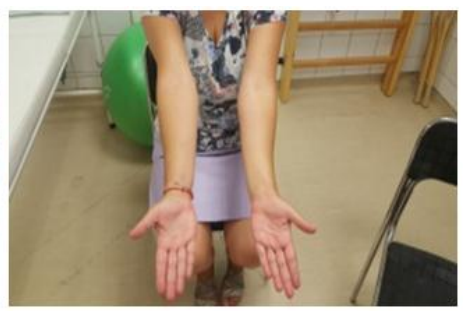

(d)

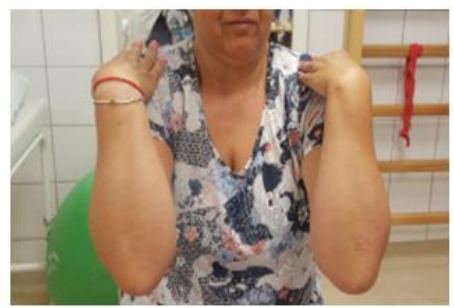

Case 2: Figures: 6 Range of motion at one year

\section{Discussion}

Simultaneous fractures of both ends of radius or equivalent combinations lesions (fracture - dislocation of radial head and distal radius) are uncommon injuries. ${ }^{[4]}$ From 2001, when Nagaya described the first case, until today seven case reports have been published internationally. ${ }^{[6,9]}$ Agarwal (2007) referred that there is no age group exempt from this bipolar injuries. The mechanism of this injury depends on whether the elbow is flexed or extended and the position of the forearm at the time of injury. Henrikson founds that individuals with increased carrying angle are predisposed to valgus stress in falls with elbow extended, resulting in fracture of proximal radius and/ulna. Usually this is the result of a compression axial force when patient falls on an outstretched hand with the elbow hyperextended. This provokes the distal radius fracture first, and then the elbow is enforced in valgus position with the force leading to the radiocapitellar joint and finally resulting in radial head fracture.

While in this concomitant double injury of the radius the fracture of distal radius is the first diagnosed in emergency room this is not happening in case of radial head injury (represented by a spectrum of variation from Maison I to IV) which might be misdiagnosed even in simple fractures. For this reason, comprehensive clinical and radiological examination of both joints of forearm should be done routinely though there is an index of suspicion of double injuries of radius. Baghel et al (2016) referred that thereby it is important to recognize its occurrence, the unusual mechanism of injury, and the management. ${ }^{[9]}$

Optional therapy of this double radius injury is not clearly described in literature. We addressed that in management of this lesion two majors factors must be considered: patient characteristics (age, physical demand of the patient) and fracture factors (fracture pattern, stability). Treatment of distal radius fracture (D.R.F.) varying from closed reduction and Plaster of Paris application to K-wire fixation, external fixation (ligamentotaxsis) and finally open reduction and volar locking plate. ${ }^{[3,4,5,6]}$ We agree that ORIF of D.R.F as a treatment option has the advantage to restore the radial height, anatomical reduction of radiocarpal joint, is a stable fixation and permits early mobilization, and therefore we addressed palmar volar locking plate in distal radius fractures. ${ }^{[6]}$

Management of radial head fracture is clearly described. ${ }^{[11]}$ When the fracture is Maison I, II (without mechanical block) conservative option is preferable, while in Maison III,IV surgical treatment is the gold of standard. Different options are available for the surgical procedure of comminuted fractures of radial head. Van Riet(2003) advocated that the radial head should be preserved by internal fixation even when the fractures are severely displaced or comminuted and the choice of implant varied from mini plates or Herbert screws or Kirschner wires. ${ }^{[14]}$ A retrospective study showed that open reduction and internal fixation is best reserved for minimally comminuted fractures of the radial head with three or fewer articular fragments. ${ }^{[13]}$ On the other side, there are proponents of primary radial head excision and replacement in comminuted fracture with the scope to prevent elbow stiffness and future proximal migration, and referred that it has always been a viable treatment option. ${ }^{[6,11]}$ In our case with Maison type III fracture, the radial head was very comminuted and it was decided to replace the radial head. At one year follow-up the patient has a painfree, functional elbow with almost full range of motion. For these reasons we suggest stable ORIF when is possible and if not, replacement with radial prosthesis is chosen with scope to prevent elbow stiffness.

The critical question arised from is, which fracture we approach first? We agree with Kamadulin, that ORIF of distal radius fracture has the advantage (when osteosynthesis of radial head fracture is not possible and the radial head must be replaced) of restoring the radial height and Lister's tubercle (two major landmarks) and help 
us to decide the length size of radial head and the correct rotation of the prosthesis.

\section{Conclusion}

Simultaneous fractures of both ends of radius are uncommon injuries. We emphasized in detailed clinical examination in all wrist injuries in the emergency with the aim that bipolar injuries of the radius must not be misdiagnosed. When surgery is inevitable the first fracture that must be restored is the distal radius one. In young patients it must be tried to preserve radial head with osteosynthesis, but not against of future elbow joint range of motion. Radial head replacement is a good treatment option with good clinical outcomes decreasing future complications of elbow joint.

\section{Conflict of Interest}

The authors declare that there is no conflict of interest. We certify that no funding has been received for the conduct of this study and/or preparation of this text.

\section{Ethics approval}

Our institution does not require ethical approval for reporting individual cases or case series.

\section{Informed consent}

Verbal informed consent was obtained from the patient(s) for their anonymized information to be published in this article.

\section{References}

1. Nellans KW, Kowalski E, Chung KC. The Epidemiology of Distal Radius Fractures. Hand Clin. 2012; 28(2):113125.https://doi.org /10.1016/j.hcl.2012. 02.001

2. Kaas L, van Riet RP, Vroemen JP, Eygendaal D. The epidemiology of radial head fractures.J shoulder Elbow Surg.2010;19(4):520-523.https://doi.org/10.1016 /j.jse.2009.10.015.
3. Agarwal A. Ipsilateral fracture of distal and proximal ends of the radius: does this injury pattern deserve special attention? Eur J Orthop Surg Traumatol. 2007; 17(2): 181-187.

4. Nagaya H, Saito Y, Warashina H. Simultaneous ipsilateral fractures of distal and proximal ends of radius. J Orthop Sci. 2001;6: 439e443.

5. El Kadi K, Benabid M, Saliou S, et al. Simultaneous ipsilateral fractures of distal and proximal ends of the radius. Pan Afr Med J. 2017; 27: 98. https://doi.org/10.11604/pamj.2017.27.98.3504.

6. Akma Kamaludin NA, Ferdaus Kamudin NAF, Abdullah S, Sapuan J. Ipsilateral proximal and distal radius fractures with unstable elbow joint: Which should we address first? Chin J. Traumatology. 2019; 22(1): 59 - 62 https://doi.org/10.1016/j.cjtee.2018.04.007

7. Cooney WP, Bussey R, Dobyns JH et al. Difficult wrist fractures: perilunate fracture-dislocations of the wrist. Clin Orthop Rel Res. 1987; 214: 136-47.

8. Yan W, Wang L, Miao J. Comminuted fractures of ipsilateral radial head and distal radius: A rare injury pattern. Chin J. Traumatol; 2015;18(2):106-8.

9. Baghel A, Agrawal A, Kushwaha NS, Singh S. Bipolar Radial fracture: A Unique injury pattern. International J. of Contemporary Medical Research. 2016;3(7):20382040.

10. Henrikson B. Isolated fracture of the proximal end of the radius in children. Acta Orthop Scand. 1969; 40(2): 246260.

11. Pappas N, Bernstein J. Fractures in Brief: Radial Head Fractures. Clin Orthop Relat Res (2010) 468:914-916, doi 10.1007/s11999-009-1183-1

12. van Riet RP, Van Glabbeek F, Neale PG, et al. The noncircular shape of the radial head. J Hand Surg Am. 2003;28:972 - 978.

13. Ring D, Quintero J, Jupiter JB. Open reduction and internal fixation of fractures of the radial head. J Bone Joint Surg Am. 2002;84-A:1811 -1815.

14. Li YA, Chang MC, Chen TS. Extreme comminuted fracture of the radial head fixed with multiple Kirschner wires: case report. J Trauma. 2009;67:124-128. 